\title{
OPTIMIZATION OF INPUT COVARIANCE MATRIX FOR MUlti-ANTENNA CORRELATEd CHANNElS
}

\author{
Feng Li \\ Department of Operation Research and Information Engineering, Cornell University, \\ Ithaca, NY, USA, 14850 \\ fl263@cornell.edu
}

\begin{abstract}
Optimizing the input covariance matrix of a multiple-antenna transmit system with partial channelstructure feedback is an important issue to fully exploit the channel capacity. Efficient design of the optimal input covariance matrix, however, remains unavailable although its eigenvector structure was clearly revealed in a recent publication. In this paper, we obtain an explicit derivative function forming a solid basis for optimizing the optimal input covariance matrix. This new derivative expression enables us to further develop an efficient iterative algorithm for determining the optimal eigenvalues. The technique is illustrated through numerical examples.
\end{abstract}

\section{KEYWORDS}

Channel capacity, multiple-input-single-output (MISO) channel, channel covariance matrix, NewtonRaphson method.

\section{INTRODUCTION}

In communication theory, MIMO refers to radio links with multiple antennas at the transmitter and the receiver side [1]-[8]. Given multiple antennas, the spatial dimension can be exploited to improve the performance of the wireless link. The performance is often measured as the average bit rate (bit/s) the wireless link can provide or as the average bit error rate (BER). Which one has most importance depends on the application. Given a MIMO channel, duplex method and a transmission bandwidth, the system can be categorized as Flat or frequency selective fading and with full, limited or without transmitter channel state information (CSI) [9]-[14].

In a TDD system with a duplex time less than the coherence time of the channel, full CSI is available at the transmitter, since then, the channel is reciprocal. In FDD systems, there commonly exists a feedback channel from the receiver to the transmitter that provides the transmitter with some partial CSI. This could be information of which subgroup of antennas to be used or which eigen-mode of the channel that is strongest. It is also possible to achieve a highly robust wireless link without any CSI at the transmitter, by using transmit diversity. Diversity can be achieved through so called space-time codes, like the Alamouti code for two transmit antennas and high bit rates is achieved by spatial multiplexing systems, such as the pioneer system from Bell Labs abbreviated as BLAST [15], [16].

If a broadband wireless connection is desired, the symbol rate must be increased further which at some point will lead to a frequency selective channel. Then, there are two ways to go, either we employ pre- or post-equalization of the channel or we divide the channel into many narrowband flat fading sub-channels, a technique utilized by OFDM, and transmit our data on these sub streams, without the need for channel equalization. Hence, it is always possible to convert a frequency selective channel to many flat fading channels using OFDM and apply the developed flat fading MIMO signalling techniques to each of these sub-channels. 
International Journal of Computer Networks \& Communications (IJCNC) Vol.3, No.5, Sep 2011

When full CSI is available at the transmitter, it is possible to transmit data on the MIMO channel eigen-modes [17], [18]. A MIMO system with $\mathrm{N}$ transmit antennas and $\mathrm{M}$ receive antennas has $\min (\mathrm{N}, \mathrm{M})$ eigen-modes. The gain of these eigen-modes is proportional to the singular values of the MIMO channel, so they have disparate power. Recently, there have been some profound contributions in delay-limited channels assuming the use of causal CSIT. In [19], Negi and Cioffi investigated the optimal power control for minimizing the outage probability using a dynamic programming (DP) approach with certain power constraints. Similar methodology was also proposed in [20] for a two user downlink channel for expected capacity maximization with a short-term power constraint. Furthermore, in [21], Berry and Gallager looked into the delay-constrained problem taking into account the size of the buffer. Most recently in [22], an algorithm that finds the optimal power allocation over the blocks to minimize the overall transmit power while constraining an upper bound of the outage probability constraint was proposed. Unfortunately, the assumption of having perfect CSIT is questionable, and the required amount of channel feedback may not justify the diversity gain obtained from the intelligent power control. Sometimes, only limited feedback, for example, statistical information of the channel, is available.

It is well known that appropriately exploiting partial channel knowledge at the transmitter can increase the information throughput of a wireless multiple-antenna system with either a MIMO [26], [27] or a multi-input single-output(MISO) [23]-[25] configuration. The case of MISO in correlated Rayleigh fading was recently tackled in [23], [25] aiming to obtain the optimal input covariance matrix for which the channel capacity is maximized. An important finding in [23] is that the optimal input covariance matrix should have the same eigenvectors as the channel covariance matrix, suggesting that independent Gaussian signals be transmitted along the direction defined by the eigenvectors of the channel covariance matrix. This finding was shortly extended to the general case with MIMO antennas [24], [34], [35].

In all aforementioned studies, however, the determination of the eigen-values for the optimal input covariance matrix relies on numerical search, directly based on the original objective function for optimization except the insertion of the optimal eigenvectors. The gradient descent method is usually used for iterative search. The drawback is inaccuracy on one hand and time consuming on the other, thereby calling for more feasible theoretic results to be used in the system design. The purpose of this paper is two-fold: to establish a simple relation that defines the optimal eigenvalues, and to derive an efficient algorithm for their evaluation.

The rest of this paper is organized as follows. In Section 2, 3, 4 and 5, we present our system model and the detailed procedure of our proposed algorithm to determine the structure of the optimum input covariance matrix. In Section 6, we present some numerical results which reveal the truth behind the selection of optimum input covariance matrix and also illustrate the efficiency of our algorithm. Finally, Section 7 contains some concluding remarks.

As a convention in this paper, we will use superscript $†$ to signify conjugate transposition, and use $E[\cdot], \operatorname{diag}\{\cdots\}$ and $\operatorname{tr}(\cdot)$ to denote expectation,the diagonal matrix and the trace of a matrix, respectively. The notation $\mathbf{x} \sim C N m(\mu, \mathbf{R})$ implies that the $m$-by-1 vector $\mathbf{x}$ is complex Gaussian distributed with mean $\mu$ and covariance matrix $\mathbf{R}$. For the case of $m=1$, the subscript $m$ will be dropped for simplicity.

\section{FORMULATION}

Consider a wireless MISO system with $m$ transmit antennas and one receive antenna. Let $\mathbf{x}$ and 
h denote the $m \times 1$ transmitted signal vector and the channel gains linking the transmit antennas to the receiver, respectively, so that the received signal $y$ can be written as

$$
y=\mathbf{x}^{\dagger} \mathbf{h}+n .
$$

where $n$ is additive white Gaussian noise with distribution

$$
n \sim C N\left(0, \sigma_{n}^{2}\right)
$$

and for Rayleigh fading, we have

$$
\mathbf{h} \sim C N_{m}\left(\mathbf{0}, \mathbf{R}_{h}\right)
$$

Suppose that partial channel information is fed back to the receiver, so that the receiver knows the Gaussianity of $\mathbf{h}$ with zero mean and covariance matrix $\mathbf{R} h$. According to Shannon, the optimal distribution of $\mathbf{x}$ that maximizes the channel capacity is the joint Gaussian distribution taking the form of

$$
\mathbf{x} \sim C N_{m}(\mathbf{0}, \mathbf{Q})
$$

The question is for given partial channel information $\mathbf{R} h$ at the receiver, how to determine the optimal covariance structure $\mathbf{Q}$ subject to the constraint of a constant transmitted power, i.e.,

$$
\operatorname{tr}\{\mathbf{Q}\}=P
$$

such that

$$
C=E_{h}\left[\log \left(1+\sigma_{n}^{-2} \mathbf{h}^{\dagger} \mathbf{Q h}\right)\right]
$$

is maximized. The authors of [1] have found that the optimal $\mathbf{Q}$ should have the same unitary matrix of eigenvectors as that of $\mathbf{R} h$. Efficient techniques, however, are not available for determining the eigenvalues of the optimal $\mathbf{Q}$ except for numerical search.

In this paper, we will employ appropriate mathematical skills to directly obtain the derivative of $C$ with respect to $\mathbf{Q}$ whereby the complete structure of the optimal $\mathbf{Q}$ can be determined, and an efficient algorithm is established. In particular, we use the Lagrange multipliers to form a new objective function, as shown by

$$
J(\mathbf{Q})=E\left[\log \left(1+\sigma_{n}^{-2} \mathbf{h}^{\dagger} \mathbf{Q h}\right)\right]+\lambda(\operatorname{tr}\{\mathbf{Q}\}-P) .
$$

where $\lambda$ is a constant. The task is to find $\mathbf{Q}$ that maximizes this objective function. 
International Journal of Computer Networks \& Communications (IJCNC) Vol.3, No.5, Sep 2011

\section{EQUATIONS FOR OPTIMAL $Q$}

To maximize $\mathcal{M} \mathbf{Q})$, we take its derivative with respect to $\mathbf{Q}$ yielding

$$
\frac{d J}{d \mathbf{Q}}=\frac{d E[C]}{d \mathbf{Q}}+\lambda \frac{\operatorname{tr}\{\mathbf{Q}\}}{d \mathbf{Q}}
$$

The second term on the right is simply equal to the identity matrix I; namely,

$$
\frac{d \operatorname{tr}\{\mathbf{Q}\}}{d \mathbf{Q}}=\mathbf{I}
$$

We therefore focus on the first term. Using the rule (10.17) for matrix differentiation [29], we obtain

$$
\begin{aligned}
\frac{d E[C]}{d \mathbf{Q}} & =E\left[\frac{d C}{d \mathbf{Q}}\right] \\
& =E\left[\frac{\mathbf{h h}^{\dagger}}{\sigma_{n}^{2}+\mathbf{h}^{\dagger} \mathbf{Q h}}\right] .
\end{aligned}
$$

On the first line, we have changed the order of expectation and differentiation since both are linear operators. The expectation is taken on the random vector $\mathbf{h}$. As we can see on the second line, however, the function inside the expectation operator contains $\mathbf{h}$ in both of its denominator and numerator. It is nearly impossible to use brute force to obtain the expected value. We therefore consider, instead, the use of the identity

$$
\frac{1}{\sigma_{n}^{2}+\mathbf{h}^{\dagger} \mathbf{Q h}}=\frac{1}{\sigma_{n}^{2}} \int_{0}^{\infty} \exp \left\{-x\left(1+\sigma_{n}^{-2} \mathbf{h}^{\dagger} \mathbf{Q h}\right)\right\} d x .
$$

to convert the denominator into an exponential whereby the expectation can be performed. The result is shown in order.

$$
\begin{aligned}
\frac{d E[C]}{d \mathbf{Q}} & =E\left[\int_{0}^{\infty} \frac{\mathbf{h h}^{\dagger}}{\sigma_{n}^{2}} \exp \left\{-x\left(1+\sigma_{n}^{-2} \mathbf{h}^{\dagger} \mathbf{Q h}\right)\right\} d x\right] \\
& =-E\left[\int_{0}^{\infty} \frac{\exp (-x)}{x} \frac{d}{d \mathbf{Q}} \exp \left(-x \sigma_{n}^{-2} \mathbf{h}^{\dagger} \mathbf{Q} \mathbf{h}\right) d x\right] \\
& =-\int_{0}^{\infty} \frac{\exp (-x)}{x} \frac{d}{d \mathbf{Q}} E\left[\exp \left(-x \sigma_{n}^{-2} \mathbf{h}^{\dagger} \mathbf{Q} \mathbf{h}\right)\right] d x
\end{aligned}
$$

The expectation on the last line is essential to determine the characteristic function of the quadratic form

$$
\sigma_{n}^{-2} \mathbf{h}^{\dagger} \mathbf{Q h}
$$


International Journal of Computer Networks \& Communications (IJCNC) Vol.3, No.5, Sep 2011

in Gaussian vector, which is known to be [30]

$$
E\left[\exp \left(-x \sigma_{n}^{-2} \mathbf{h}^{\dagger} \mathbf{Q h}\right)\right]=\operatorname{det}\left(\mathbf{I}+x \sigma_{n}^{-2} \mathbf{R}_{h} \mathbf{Q}\right)^{-1}
$$

Insert (14) into (12) yielding

$$
\begin{aligned}
& \frac{d E[C]}{d \mathbf{Q}} \\
& =-\int_{0}^{\infty} \frac{\exp (-x)}{x} \frac{d}{d \mathbf{Q}} \operatorname{det}\left(\mathbf{I}+x \sigma_{n}^{-2} \mathbf{R}_{h} \mathbf{Q}\right)^{-1} d x \\
& =-\int_{0}^{\infty} \frac{\exp (-x) d\left(\operatorname{det}\left(\mathbf{I}+x \sigma_{n}^{-2} \mathbf{R}_{h} \mathbf{Q}\right)^{-1}\right)}{\sigma_{n}^{2} \mathbf{R}_{h}^{-1} d\left(\mathbf{I}+x \sigma_{n}^{-2} \mathbf{R}_{h} \mathbf{Q}\right)} d x
\end{aligned}
$$

which, after invoking the formula for matrix differentiation [29]

$$
\frac{d \operatorname{det}\left(\mathbf{A}^{n}\right)}{d \mathbf{A}}=n \operatorname{det}\left(\mathbf{A}^{n}\right)\left(\mathbf{A}^{-1}\right)^{\dagger}
$$

and simplifying, produces

$$
\begin{aligned}
\frac{d E[C]}{d \mathbf{Q}} & =\int_{0}^{\infty} \frac{\exp (-x) \mathbf{R}_{h}\left[\left(\mathbf{I}+x \sigma_{n}^{-2} \mathbf{R}_{h} \mathbf{Q}\right)^{-1}\right]^{\dagger}}{\sigma_{n}^{2} \operatorname{det}\left(\mathbf{I}+x \sigma_{n}^{-2} \mathbf{R}_{h} \mathbf{Q}\right)} d x \\
& =\int_{0}^{\infty} \frac{\left.\exp (-x)\left[\mathbf{R}_{h}^{-1}+x \sigma_{n}^{-2} \mathbf{Q}\right)^{-1}\right]^{\dagger}}{\sigma_{n}^{2} \operatorname{det}\left(\mathbf{I}+x \sigma_{n}^{-2} \mathbf{R}_{h} \mathbf{Q}\right)} d x .
\end{aligned}
$$

By inserting (17) into (8) and setting the derivative to zero, we obtain the simultaneous Equations

$$
\mathbf{A}+\lambda \mathbf{I}=\mathbf{0}
$$

where $\mathbf{A}$ is a matrix function of $\mathbf{Q}$ defined by

$$
\mathbf{A}=\int_{0}^{\infty} \frac{\exp (-x)\left[\left(\mathbf{R}_{h}^{-1}+x \sigma_{n}^{-2} \mathbf{Q}\right)^{-1}\right]^{\dagger}}{\sigma_{n}^{2} \operatorname{det}\left(\mathbf{I}+x \sigma_{n}^{-2} \mathbf{R}_{h} \mathbf{Q}\right)} d x
$$

The solution to (18) defines the optimal $\mathbf{Q}$.

\section{Optimal Solution}

Let $\mathbf{U} 1$ denote the matrix of eigenvectors of $\mathbf{R} h$ and let

$$
\boldsymbol{\Lambda}=\operatorname{diag}\left\{\alpha_{1}, \alpha_{2}, \cdots, \alpha_{m}\right\}
$$

denote its corresponding matrix of eigenvalues. In a similar manner, we define the eigen 
structure of $\mathbf{Q}$ by $\mathbf{U} 2$ and $\mathbf{\Omega}$. Hence, we can write

$$
\begin{aligned}
\mathbf{R}_{h} & =\mathbf{U}_{1} \boldsymbol{\Lambda} \mathbf{U}_{1}^{\dagger} \\
\mathbf{Q} & =\mathbf{U}_{2} \boldsymbol{\Omega} \mathbf{U}_{2}^{\dagger}
\end{aligned}
$$

where

$$
\boldsymbol{\Omega}=\left\{\beta_{1}, \beta_{2}, \cdots, \beta_{m}\right\}
$$

From (18), it follows that the optimal $\mathbf{Q}$ must be chosen such that $\mathbf{A}$ is, up to a factor, the identity matrix. This requires, in turn, that all the eigenvalues of $\mathbf{A}$ be identical. The optimal $\mathbf{Q}$ that meets this requirement implies that

$$
\begin{aligned}
& \mathbf{U}_{2}=\mathbf{U}_{1}=\mathbf{U} \\
& y_{1}=y_{2}=\cdots=y_{m} .
\end{aligned}
$$

where for $i=1, \ldots, m$,

$$
y_{i}=\int_{0}^{\infty} \frac{\exp (-x)\left(\alpha_{i}^{-1}+x \sigma_{n}^{-2} \beta_{i}\right)^{-1}}{\sigma_{n}^{2} \prod_{i=1}^{m}\left(1+x \sigma_{n}^{-2} \alpha_{i} \beta_{i}\right)} d x
$$

These results can be easily verified if we use the relation

$$
\mathbf{A}=\mathbf{U} \operatorname{diag}\left\{y_{1}, y_{2}, \cdots, y_{m}\right\} \mathbf{U}^{\dagger}
$$

to represent (18) as

$$
\mathbf{U}\left[\operatorname{diag}\left\{y_{1}, y_{2}, \cdots, y_{m}\right\}+\lambda \mathbf{I}\right] \mathbf{U}^{\dagger}=\mathbf{0} .
$$

It requires that

$$
y_{1}+\lambda=y_{2}+\lambda=\cdots=y_{m}+\lambda=0
$$

leading to the result shown in the second line of (23). Besides revealing the same eigenvectors of $\mathbf{Q}$ as described in [23], our results also establish basic relation among the eigenvalues of $\mathbf{Q}$.

The relation among $\left\{y_{i}\right\}$, plus the power constraint, defines the following simultaneous equations

$$
\begin{aligned}
& y_{1}-y_{2}=0 \\
& y_{1}-y_{3}=0 \\
& \vdots \\
& y_{1}-y_{m}=0 \\
& \beta_{1}+\cdots \beta_{m}-P=0 .
\end{aligned}
$$

which is a set of nonlinear equations in the eigenvalues $\left\{\beta_{i}\right\}$ of the optimal $\mathbf{Q}$. 
International Journal of Computer Networks \& Communications (IJCNC) Vol.3, No.5, Sep 2011

\section{EFFiCIENT Algorithm}

In this section, we develop an iterative algorithm for solving the nonlinear equations in (28), based on the Newton-Raphson method [31]. The recursive algorithm so obtained is capable to rapidly approach the optimal solution, at the rate of geometric convergence. Define vectors

$$
\begin{aligned}
\beta & =\left(\begin{array}{c}
\beta_{1} \\
\beta_{2} \\
\vdots \\
\beta_{m}
\end{array}\right) \\
F\left(\beta^{k}\right) & =\left(\begin{array}{c}
f_{1}\left(\beta^{k}\right) \\
f_{2}\left(\beta^{k}\right) \\
\vdots \\
f_{m}\left(\beta^{k}\right)
\end{array}\right) .
\end{aligned}
$$

where

$$
\begin{aligned}
& f_{1}=y_{1}-y_{2} \\
& \vdots \\
& f_{m-1}=y_{1}-y_{m} \\
& f_{m}=\beta_{1}+\cdots \beta_{m}-P .
\end{aligned}
$$

Here, the superscript $k$ is used to signify the results obtained at the $k$ th iteration. We determine the Jacobian matrix $[\mathcal{J}(\beta k)]$ by taking the derivatives of $\left\{f_{i}\right\}$ with respect to $\left\{\beta_{j}\right\}$. The result is given by

$$
\left[J\left(\beta^{k}\right)\right]=\left(\begin{array}{cccc}
\frac{\partial f_{1}}{\partial \beta_{1}} & \frac{\partial f_{1}}{\partial \beta_{2}} & \cdots & \frac{\partial f_{1}}{\partial \beta_{m}} \\
\vdots & \vdots & \ddots & \vdots \\
\frac{\partial f_{m-1}}{\partial \beta_{1}} & \frac{\partial f_{m-1}}{\partial \beta_{2}} & \cdots & \frac{\partial f_{m-1}}{\partial \beta_{m}} \\
1 & 1 & \cdots & 1
\end{array}\right)
$$

where

$$
\begin{gathered}
\frac{\partial f_{i}}{\partial \beta_{1}}=\int_{0}^{\infty} \frac{\left(\alpha_{1} \alpha_{i+1}\left(\beta_{1}-2 \beta_{i+1}\right) \sigma_{n}^{-2} x+\left(\alpha_{i+1}-2 \alpha_{1}\right)\right) \alpha_{1} d x}{\left(1+x \sigma_{n}^{-2} \alpha_{1} \beta_{1}\right)^{2}\left(1+x \sigma_{n}^{-2} \alpha_{i+1} \beta_{i+1}\right) \eta} \\
\frac{\partial f_{i}}{\partial \beta_{i+1}}=\int_{0}^{\infty} \frac{\left(\alpha_{1} \alpha_{i+1}\left(2 \beta_{1}-\beta_{i+1}\right) \sigma_{n}^{-2} x+\left(2 \alpha_{i+1}-\alpha_{1}\right)\right) \alpha_{i+1} d x}{\left(1+x \sigma_{n}^{-2} \alpha_{i+1} \beta_{i+1}\right)^{2}\left(1+x \sigma_{n}^{-2} \alpha_{1} \beta_{1}\right) \eta} \\
\frac{\partial f_{i}}{\partial \beta_{j}}=\int_{0}^{\infty} \frac{\left(\alpha_{1} \alpha_{i+1}\left(\beta_{1}-\beta_{i+1}\right) \sigma_{n}^{-2} x+\left(\alpha_{i+1}-\alpha_{1}\right)\right) \alpha_{j} d x}{\left(1+\alpha_{1} \beta_{1} \sigma_{n}^{-2} x\right)\left(1+\alpha_{i+1} \beta_{i+1} \sigma_{n}^{-2} x\right)\left(1+\alpha_{j} \beta_{j} \sigma_{n}^{-2} x\right) \eta} \\
i=1, \ldots, m-1, \quad j=2, \ldots, m, \quad j \neq i+1 .
\end{gathered}
$$


with $\eta$ denoting

$$
\eta=\frac{\sigma_{n}^{4} \exp (x)}{x} \prod_{l=1}^{m}\left(1+x \sigma_{n}^{-2} \alpha_{l} \beta_{l}\right) .
$$

The solution to the eigenvalues of the optimal $\mathbf{Q}$ can be obtained by performing the following iteration:

$$
\beta^{k+1}=\beta^{k}-\left[J\left(\beta^{k}\right)\right]^{-1} F\left(\beta^{k}\right) .
$$

which usually converges in a few steps.

TABLE I

THE OPTIMUM EIGENVALUE RESULTS FOR TWO TRANSMIT ELEMENTS

\begin{tabular}{|r|c|c|c|}
\hline$\alpha_{1}: \alpha_{2}$ & $\beta_{1}$ & $\beta_{2}$ & channel capacity $(\mathrm{bps} / \mathrm{Hz})$ \\
\hline $1: 1$ & $0.5 \mathrm{P}$ & $0.5 \mathrm{P}$ & 3.6791 \\
$2: 1$ & $0.71 \mathrm{P}$ & $0.29 \mathrm{P}$ & 3.7267 \\
$3: 1$ & $0.82 \mathrm{P}$ & $0.18 \mathrm{P}$ & 3.7897 \\
$4: 1$ & $0.9 \mathrm{P}$ & $0.1 \mathrm{P}$ & 3.8461 \\
$5: 1$ & $0.95 \mathrm{P}$ & $0.05 \mathrm{P}$ & 3.8907 \\
$\geq 5.93: 1$ & $\mathrm{P}$ & 0 & 3.9310 \\
\hline
\end{tabular}

TABLE II

THE OPTIMUM EIGENVALUE RESULTS FOR THREE TRANSMIT ELEMENTS

\begin{tabular}{|r|c|c|c|c|}
\hline$\alpha_{1}: \alpha_{2}: \alpha_{3}$ & $\beta_{1}$ & $\beta_{2}$ & $\beta_{3}$ & channel capacity $(\mathrm{bps} / \mathrm{Hz})$ \\
\hline $1: 1: 1$ & $0.33 \mathrm{P}$ & $0.33 \mathrm{P}$ & $0.33 \mathrm{P}$ & 3.3741 \\
$2: 1: 1$ & $0.648 \mathrm{P}$ & $0.176 \mathrm{P}$ & $0.176 \mathrm{P}$ & 3.4650 \\
$2: 2: 1$ & $0.488 \mathrm{P}$ & $0.488 \mathrm{P}$ & $0.024 \mathrm{P}$ & 3.4620 \\
$3: 1: 1$ & $0.8 \mathrm{P}$ & $0.1 \mathrm{P}$ & $0.1 \mathrm{P}$ & 3.5775 \\
$3: 2: 1$ & $0.62 \mathrm{P}$ & $0.38 \mathrm{P}$ & 0 & 3.5199 \\
$8: 1: 1$ & $\mathrm{P}$ & 0 & 0 & 3.8642 \\
\hline
\end{tabular}

Let us summarize the procedure for the determination of $\mathbf{Q}$.

a) Eigen decompose $\mathbf{R} h$ to obtain its matrix of eigenvector $\mathbf{U}$ and eigenvalues $\left\{\alpha_{1}, \ldots, \alpha_{m}\right\}$.

b) Use the values of $\left\{\alpha_{i}\right\}$, along with $\left\{\beta_{i} k\right\}$ obtained at each iteration, to (24) and (31) to determine $\left\{y_{i}\right\}$ and $\{f i\}$.

c) Calculate the Jacobian matrix using (32).

d) Update the eigenvalue using (35).

Once we obtain the optimal eigenvalues, we can use it, along with the optimal eigenvectors to determine the maximum average capacity using (6). This type calculation is needed in the next section for numerical illustration. To simplify the evaluation, it is easy to show that (6) is statistically equivalent to

$$
E[C]=E\left[\log \left(\sum_{i=1}^{m} \frac{\alpha_{i} \beta_{i}}{2 \sigma^{2}} \gamma_{i}+1\right)\right], \quad \gamma_{i} \sim \chi^{2}(2) .
$$


Where

$$
\left\{\gamma_{i}, i=1, \cdots, m\right\}
$$

are independent chi-square variables with 2 degrees of freedom. The distribution function of $y_{i}$ can be explicitly written as

$$
f_{2}\left(\gamma_{i}\right)=\frac{\exp \left(-\frac{\gamma_{i}}{2}\right)}{2}
$$

\section{NUMERICAL RESULTS}

To further reveal the truth behind the selection of optimum input covariance matrix, we take two
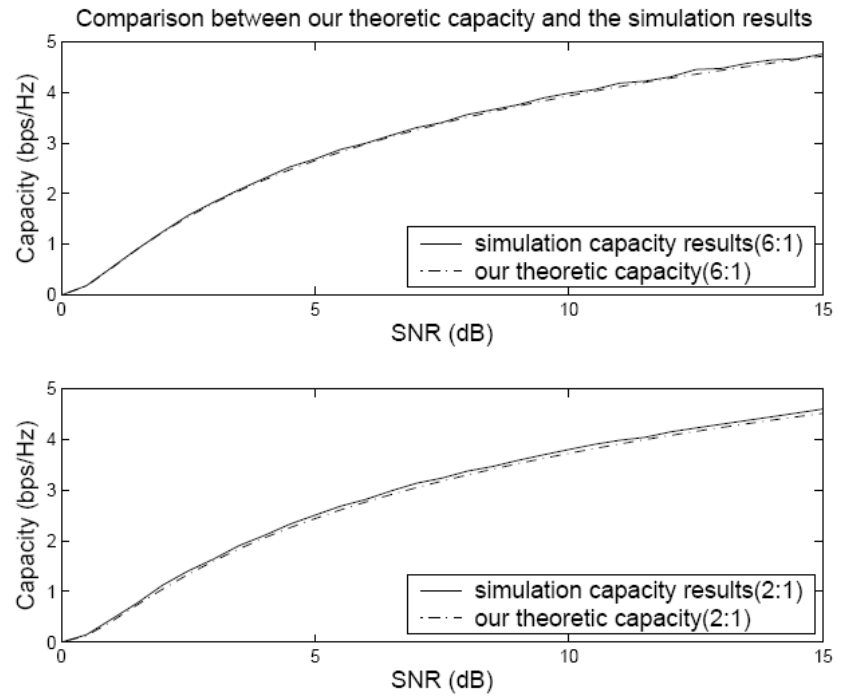

Fig. 1. Our theoretical capacity compared with the simulation capacity results for two transmit elements, where (a:b) means $\alpha_{1}: \alpha_{2}=a: b$.

and three transmit elements for example respectively. Here we suppose $\sigma_{n}^{2}=1, P=10$, therefore $S N R=10 \log P / \sigma_{n}^{2}=10 \mathrm{~dB}$.

When $m=2$, by our proposed optimization method, we have the following results: When $\alpha_{1}=\alpha$ 2, $\beta_{1}=\beta_{2}=0.5 P$. When $\alpha_{1}=2 \alpha_{2}, \beta_{1}=0.71 P, \beta_{2}=0.29 P$. When $\alpha_{1}=3 \alpha_{2}, \beta_{1}=0.82 P, \beta_{2}=$ $0.18 P$. When $\alpha_{1}=4 \alpha_{2}, \beta_{1}=0.9 P, \beta_{2}=0.1 P$. When $\alpha_{1}=5 \alpha_{2}, \beta 1=0.95 P, \beta_{2}=0.05 P$. When $\alpha_{1}>$ $5.93 \alpha_{2}, \beta 1=P, \beta_{2}=0$. Similarly, when $m=3$, by our proposed optimization method, we have: When $\alpha_{1}=\alpha_{2}=\alpha_{3}, \beta_{1}=\beta_{2}=\beta_{3}=P / 3$. When $\alpha_{1}=2 \alpha_{2}=2 \alpha_{3}, \beta_{1}=0.648 P, \beta_{2}=\beta_{3}=0.176 P$. When $\alpha_{1}=\alpha_{2}=2 \alpha_{3}, \beta_{1}=\beta_{2}=0.488 P, \beta_{3}=$ 
$0.024 P$. When $\alpha_{1}=3 \alpha_{2}=3 \alpha_{3}, \beta_{1}=0.8 P, \beta_{2}=\beta_{3}=0.1 P$. When $\alpha_{1}=1.5 \alpha_{2}=3 \alpha_{3}, \beta_{1}=0.62 P, \beta_{2}$ $=0.38 P, \beta_{3}=0$. When $\alpha_{1}=8 \alpha_{2}=8 \alpha_{3}, \beta_{1}=P, \beta_{2}=\beta_{3}=0$. Table 1 and 2 illustrate this.

From the results, we can see that when the channel covariance matrix has a moderate eigenvalue spread, the eigenvectors corresponding to larger eigenvalues of channel covariance matrix should receive more power. Otherwise, when some eigenvalues are much smaller than the other eigenvalues, the optimum transmit strategy is to distribute all the power proportionally along the eigenvectors corresponding to the larger eigenvalues. In particular, when one of the eigenvalues is much larger than other ones, the best transmit strategy is to put all the power along the eigenvector corresponding to this largest eigenvalue.

In order to examine the accuracy of our method proposed above, we compare the theoretical capacity achieved by our method with the simulation capacity. For the theoretical capacity, we

substitute $\alpha_{i}$, which is supposed to have been known from the feedback, and $\beta$, which is derived by our proposed algorithm, to (25), (26). For the simulation capacity, since the eigenvectors of the optimum covariance matrix $\mathbf{Q}$, by proposition 1 , coincide with the eigenvectors of $\mathbf{R} h$, without loss of generality, the result is based on Monte Carlo calculation by using (26). For each value of $S N R$, the corresponding capacity is defined to be the largest $E[C]$
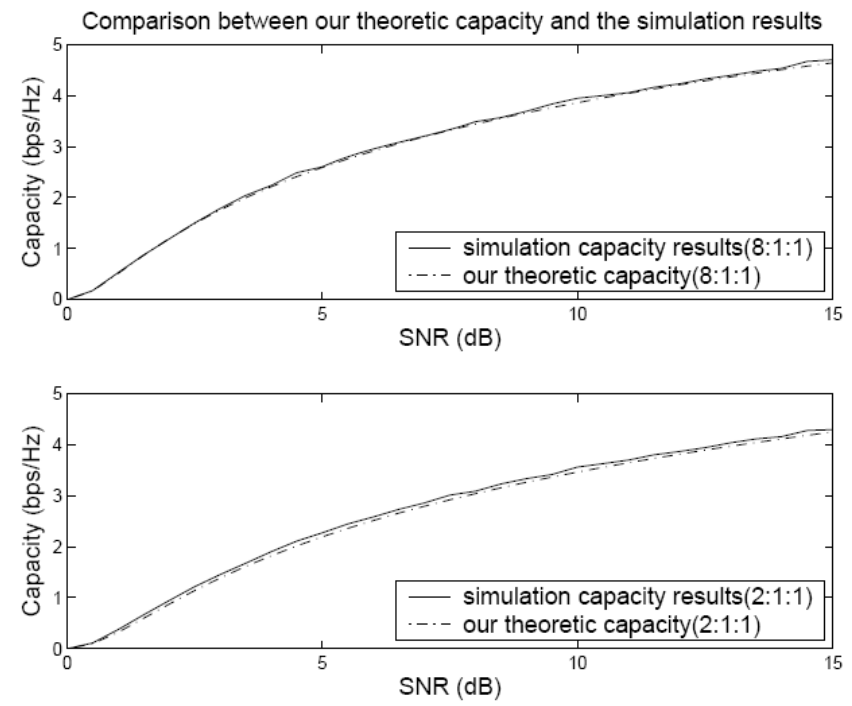

Fig. 2. Our theoretical capacity compared with the simulation capacity results for three transmit elements, where (a:b:c) means $\alpha_{1}: \alpha_{2}: \alpha_{3}=a: b: c$.

when $\beta$ itakes all the values from 0 to $P$ with step length $0.01 P$ and each value of $E[C]$ is obtained by averaging over 1000 independent computer trials.

From figure 1, 2, 3, you can see the capacity achieved by our proposed algorithm is very close to the simulation capacity result, which therefore illustrates the high accuracy of the algorithm.

\section{Conclusions}

In this paper, we propose an efficient algorithm which combines Lagrange multiplier, matrix differentiation and recursive iteration for searching the optimal input covariance matrix 
International Journal of Computer Networks \& Communications (IJCNC) Vol.3, No.5, Sep 2011

maximizing the MISO channel capacity. By our method, once we know the channel covariance matrix $\mathbf{R} h$ from feedback, we could completely find out all the eigenvalues of the optimum covariance matrix $\mathbf{Q}$ of input distribution and thus determine the exact structure of the optimum Q. Based on our algorithm, we calculate the eigenvalues of the optimum input covariance matrix for two and three transmit elements as example and find the general rule that when the channel covariance matrix $\mathbf{R} h$ has a moderate eigenvalue spread, the eigenvector of channel covariance matrix $\mathbf{R} h$ corresponding to larger eigenvalue should receive more power. However, when some eigenvalues are much smaller than the other eigenvalues, the optimum transmit strategy is to distribute all the power proportionally along the eigenvectors corresponding to the larger eigenvalues. Finally, to examine the accuracy of our method, we compare the theoretical capacity achieved by our proposed algorithm with the simulation capacity and good agreement is observed.

\section{REFERENCE}

[1] P. J. Smith and M. Shafi, "On a Gaussian approximation to the capacity of wireless MIMO systems," in Proceedings of IEEE International Conference on Communications (ICC 02), vol. 1, pp. 406-410, New York, NY, USA, May 2002.

[2] M. Kang and M.-S. Alouini, "Largest eigenvalue of complex wishart matrices and performance analysis of MIMO MRC systems," IEEE Journal on Selected Areas in Communications, vol. 21, no. 3, pp. 418-426, 2003.

[3] W. Weichselberger, M. Herdin, H. Ozcelik, E. Bonek, "A Stochastic MIMO Channel Model With Joint Correlation of Both Link Ends," IEEE Trans. Wireless Commun., vol. 5, no. 1, pp. 90-99, Jan. 2006.

[4] A. S. Y. Poon, R. W. Brodersen, D. N. C. Tse, "Degrees of Freedom in Multiple-Antenna Channels: A Signal
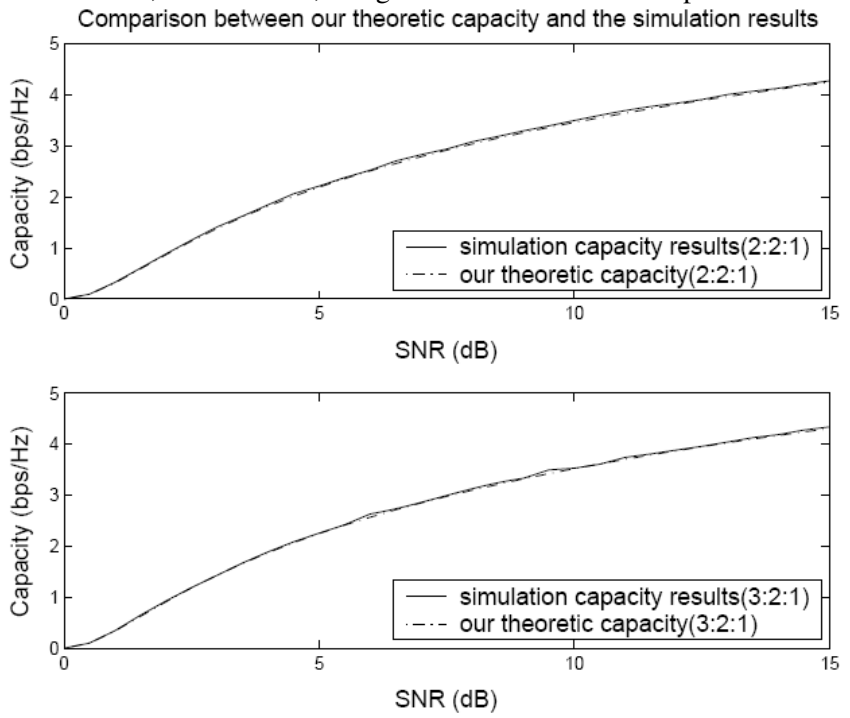

Fig. 3. Our theoretical capacity compared with the simulation capacity results for three transmit elements, where (a:b:c) means $\alpha_{1}: \alpha_{2}: \alpha_{3}=a: b: c$.

Space Approach,” IEEE Trans. Inform. Theory, vol. 51, no. 2, pp. 523-536, Feb. 2005.

[5] V. V. Veeravalli, Y. Liang, A. M. Sayeed, "Correlated MIMO Rayleigh fading channels: Capacity, Optimal Signaling, and Scaling Laws," IEEE Trans. Inform. Th., June 2005.

[6] M. Chiani, M. Z. Win, and A. Zanella, "On the capacity of spatially correlated MIMO Rayleighfading channels," IEEE Trans. Inform. Theory, vol. 49, no. 10, pp. 2363-2371, Oct. 2003.

[7] F. Li and H. Jafarkhani, "Multiple-antenna interference cancellation and detection for two users using precoders," IEEE Journal of Selected Topics in Signal Processing, vol. 3, issue 6, pp. 1066-1078, Dec. 2009.

[8] F. Li and H. Jafarkhani, "Interference cancellation and detection for more than two users," IEEE Transactions on Communications, vol. 59, issue 3, pp. 901-910, Mar. 2011.

[9] S. Jin, X. Q. Gao, and X. H. You, "On the ergodic capacity of rank-1 Ricean fading MIMO channels," IEEE Trans. Inform. Theory, vol. 53, no. 2, pp. 502-517, Feb. 2007. 
International Journal of Computer Networks \& Communications (IJCNC) Vol.3, No.5, Sep 2011

[10] M. R. McKay and I. B. Collings, "General capacity bounds for spatially correlated Rician MIMO channels," IEEE Trans. Inform. Theory, vol. 51, no. 9, pp. 3121-3145, Sep. 2005.

[11] F. Li and H. Jafarkhani, "Multiple-antenna interference cancellation and detection for two users using quantized feedback," IEEE Transactions on Wireless Communication, vol. 10, no. 1, pp. 154-163, Jan 2011.

[12] F. Li and H. Jafarkhani, "Interference cancellation and detection for multiple access channels with four users," in Proceedings of IEEE International Conference on Communications(ICC 2010), June 2010.

[13] M. Kiessling, J. Speidel, I. Viering, and M. Reinhardt, "A closed-form bound on correlated MIMO channel capacity," in Proc. IEEE VTC 2002-Fall, Vancouver, Canada, vol. 2, Sep. 2002, pp. 859-863.

[14] F. Li and Q. T. Zhang, "Transmission strategy for MIMO correlated rayleigh fading channels with mutual coupling," in Proceedings of IEEE International Conference on Communications (ICC 2007), pp. 1030-1035, June, 2007.

[15] J. P. Kermoal, L. Schumacher, K. I. Pedersen, and P. Mogensen, "A stochastic MIMO radio channel model with experimental validation,” IEEE J. Select. Areas Commun., vol. 20, no. 6, pp. 1211-1226, Jun. 2002.

[16] W. Weichselberger, M. Herdin, H. Ozcelik, and E. Bonek, "A stochastic MIMO channel model with join correlation of both link ends," IEEE Trans. Wireless Commun., vol. 5, no. 1, pp. 90-100, Jan. 2006.

[17] F. Li and H. Jafarkhani, "Using quantized feedback to cancel interference in multiple access channels," in Proceedings of IEEE Global Telecommunications Conference(Globecom 2010), December, 2010.

[18] F. Li and H. Jafarkhani, "Interference cancellation and detection using precoders", in Proceedings of IEEE International Conference on Communications (ICC 2009), June, 2009.

[19] R. Negi, and J. Cioffi, "Delay-constrained capacity with causal feedback," IEEE Trans. Info. Theory, vol. 48, no. 9, pp. 2478-2494, Sep. 2002.

[20] X. Liu, and A. J. Goldsmith, "Optimal power allocation over fading channels with stringent delay constraints," in Proc. Int. Conf. Commun., pp. 1413-1418, New York, 2002.

[21] R. Berry, and R. Gallager, "Communication over fading channels with delay constraints," IEEE Trans. Info. Theory, vol. 48. no.5, pp. 1135-1149, May 2002.

[22] K. K. Wong, "Optimizing the power allocation for Rayleigh block-fading channels with outage capacity constraints," IEEE Trans. Wireless COmmunication, vol. 6. no.9, pp. 3163-3169, Sep. 2007.

[23] E. Visotsky and U. Madhow, "Space-time transmit precoding with imperfect feedback," IEEE Trans. Inform. Theory, vol. 47, pp. 2632-2639, Sept. 2001.

[24] S. A. Jafar and A. Goldsmith, "On optimality of beamforming for multiple antenna systems," in Proc. IEEE Int. Symp. Information Theory, Washington, DC, June 2001, p. 321.

[25] A. Narula et al., "Eifficient use of side information in multiple-antenna data transmission over fading channels," IEEE J. Select. Areas Commun., vol.16, pp. 1423-1436, Oct. 1998.

[26] A. L. Moustakes et al., "Communication through a diffusive medium: Coherence and capacity," Science, vol. 287, pp. 287-290, Jan. 2000

[27] A. M. Sengupta and P. P. Mitra, "Capacity of multivariate channels with multiplicative noise: I. Random matrix technique and large-n expansions for full tranfer matrices," LANL arXiv: physics/0010081, Oct. 2000.

[28] E. Telatar, "Capacity of multi-antenna Gaussian channels," AT\&T-Bell Labs, Internal Tech. Memo, June 1995.

[29] W. H. Beyer, CRC Standard Mathematical Tables and Formulae, 29th ed. Boca Raton, FL: CRC, 1991.

[30] K.S. Miller, Complex Stochastic Processes: An Introduction to Theory and Application, Reading Massachusetts: Addison-Wesley, 1974.

[31] Mariesa Crow, Computation Methods for Electric Power System, New York: CRC Press, 2003.

[32] Robb J. Muirhead. Aspects of Multivariate Statistical Aspects. New York: Wiley, 1982.

[33] S. Jafar, S. Vishwanath, and A. Goldsmith, "Channel Capacity and beamforming for multiple transmit and receive antennas with covariance feedback", in Proc. IEEE Int. Conf. Commun., vol. 7, June 2001, pp. 22662270.

[34] S. H. Simon and A. L. Moustakas, "Optimizing MIMO systems with channel covariance feedback," IEEE J. Select. Areas Commun., vol. 21, pp. 406-417, Apr. 2003.

[35] T. Yoo, E. Yoon, and A. Goldsmith, "MIMO capacity with channel uncertainty: does feedback help?," IEEE Globecom'04, vol. 1, pp. 96-100, Dec. 2004. 